\title{
AN ELASTIC FINITE DISPLACEMENT ANALYSIS OF PLANE BEAMS WITH AND WITHOUT SHEAR DEFORMATION
}

\author{
By Taweep CHAISOMPHOB*, Fumio NISHINO**, Akio HASEGAWA*** and \\ ALY GAMAL ALY Abdel-Shafy****
}

\begin{abstract}
A finite displacemtment theory is developed for an arbitrary plane curved Timoshenko beam, in which an elastic constitutive relation is defined not by tensor components of stress and strain but by other physical components. This selection of components makes the governing equation simple and easy to handle. The nonlinear stiffness equation, with the use of nodal positions and the appropriate selection of local coordinates, is formulated for an elastic plane straight beam element. Three numerical examples of plane beam problems, involving geometrical nonlinearity, are employed as illustrative examples.
\end{abstract}

\section{INTRODUCTION}

An elastic finite displacement theory of the so-called curved Timoshenko beam with shear deformation has been studied by Reissner ${ }^{1)}$, in which the derivation of kinematic field applicable to one dimensional beam theory was a major subject of investigation. Sheinman has also studied the same subject ${ }^{2)}$. The governing equation derived in his study, howver, is applicable only for a range of moderately small rotation and in addition inconsistency seems to be present in the treatment of higher order terms of small quantities $^{3)}$. Recently, the governing equation of Timoshenko straight beams in finite deformation which is identical to that by Reissner has been formulated by Iwakuma et $\mathrm{al}^{4)}$.

For discrete system, a total Lagrangian nonlinear formulation of elastic trusses has been made by Nishino et $a^{5)}$. In their study, the stiffness equation was described as the relation between the overall nodal forces and positions, and was solved numerically by the successive substitution procedure, which was proved to be the same as Newton-Raphson iteration due to the equivalence between the total and tangential stiffness matrices of the same system.

This study presents a finite displacement theory of plane elastic beams in the range of small strain problems, and it can be divided into two parts. The first part is concerned with the formulation of a finite displacement theory of arbitrary plane curved Timoshenko beams, in which no limitation is imposed for the magnitude of displacements and rotations. It is emphasized that employing physical stress and strain components rather than tensor components simplifies the expression of constitutive equation and hence the resulting governing equation. The second part covers a total Lagrangian formulation of a plane straight

* Student Member of JSCE, M. Eng., Graduate Student, Dept. of Civil Eng., Univ. of Tokyo (Bunkyo-ku, Tokyo).

** Member of JSCE, Ph. D., Prof., Vice-President for Academic Affairs, Asian Institute of Technology (Bangkok, Thailand) on leave from University of Tokyo.

*** Member of JSCE, Dr. Eng., Asso. Prof., Dept. of Civil Eng., Univ. of Tokyo (Bunkyo-ku, Tokyo).

**** Asst. Prof., Dept. of Civil Eng., Assiut Univ. (Assiut, Egypt). 
beam element, in which its treatment is obviously easier than that of an arbitrary curved beam element. The stiffness equation, applicable for beams with and without consideration of shear deformation, is established. By utilizing nodal positions as basic unknowns and selecting the local coordinates along the displaced beam axis in the stiffness equation, the total stiffness matrix is proved to be approximately equal to the tangential stiffness matrix ${ }^{6)}$. Hence, in order to employ Newton-Raphson procedure for solving nonlinear stiffness equation, the effort to obtain tangential stiffness matrix may be ignored. Three numerical examples are presented to show the accuracy of the stiffness equation compared with the results available in literature.

\section{FORMULATION OF ARBITRARY PLANE CURVED TIMOSHENKO BEAM}

\section{(1) Kinematic Fields}

Define Cartesian coordinates $(x, y, z)$ with a reference frame of mutually orthogonal unit vectors $\left(i_{x}, i_{y}, i_{z}\right)$ fixed in space. At initial state, consider convected curvilinear coordinate $x z$ coinciding with the axis of a curved beam in $x z$ plane and straight coordinate $\eta$ perpendicular to $\xi$.

The position vector of a general point $(\xi, \eta)$ in a beam element before displacement is expressed as shown in Fig. 1 by

${ }^{0} \boldsymbol{r}(\xi, \eta)={ }^{0} \boldsymbol{r}(\xi, 0)+\eta^{0} \boldsymbol{i}_{\eta}(\xi)$

For simplicity of writing, the quantities on the beam axis are written, henceafter whenever appropriate, omitting functional relations such as ${ }^{0} \boldsymbol{r}$ and ${ }^{0} \boldsymbol{i}_{\xi}$ for ${ }^{0} \boldsymbol{r}(\xi, 0)$ and ${ }^{0} \boldsymbol{i}_{\xi}(\xi)$.

Note that the geometrical relations between the tangential and normal unit vectors along the beam axis are given as

$$
\begin{aligned}
& \frac{d^{0} \boldsymbol{r}}{d \xi}={ }^{0} \boldsymbol{i}_{\xi}, \frac{d^{0} \boldsymbol{r}}{d \eta}={ }^{0} \boldsymbol{i}_{n} \\
& \frac{d^{0} \boldsymbol{i}_{\xi}}{d \xi}=-\frac{{ }^{0} \boldsymbol{i}_{n}}{{ }^{0} \rho}, \frac{d^{0} \boldsymbol{i}_{n}}{d \xi}=\frac{{ }^{0} \boldsymbol{i}_{\xi}}{{ }^{0} \rho} \text { in which } \quad{ }^{0} \rho= \pm\left|\frac{d^{20} \boldsymbol{r}}{d \xi^{2}}\right|^{-1}
\end{aligned}
$$

The kinematic assumptions of the so-called Timoshenko beam are that plane sections normal to the beam axis before displacement remain plane but not necessarily normal after displacement and that the cross-sectional shape and area remain unchanged. The position vector of a general point at a displaced position is expressed as observed in Fig. 1 by

$$
\boldsymbol{r}(\xi, \eta)=(x+\eta \sin \Omega) \boldsymbol{i}_{x}+(z+\eta \cos \Omega) \boldsymbol{i}_{z} \text { in which } \Omega=\beta+\lambda
$$

where $\Omega$ is a rotation of cross-section measured counterclockwise form $z$-axis, $\beta$ is a shear angle, and $\lambda$ is an angle between beam axis after displacement and $\boldsymbol{i}_{x}$ measured counterclockwise, which can be defined by

$$
\begin{aligned}
& \lambda=\tan ^{-1}\left(-z^{\prime} / x^{\prime}\right)=\sin ^{-1}\left(-z^{\prime} / g\right)=\cos ^{-1}\left(x^{\prime} / g\right), \\
& g=\sqrt{\left(x^{\prime}\right)^{2}+\left(z^{\prime}\right)^{2}} \ldots \ldots \ldots \ldots \ldots \ldots \ldots \ldots \ldots \ldots \ldots \ldots \ldots \ldots \ldots \ldots \ldots \ldots \ldots \ldots \ldots
\end{aligned}
$$

in which prime indecates the differentiation with respect to $\xi$.

Define ${ }^{0} \boldsymbol{g}_{i}, \boldsymbol{g}_{i}(i=\xi, \eta)$ as the base vectors in the direction $i$ before and after displacement, respectively. Utilizing Green strain tensor $e_{i j}$ which can be defined as $\left(g_{i} g_{j}-{ }^{0} g_{i}{ }^{0} g_{j}\right) / 2$, and remembering the position field Eqs. (1-4) result in the relations between non-zero strain tensors and positions as

$$
\begin{aligned}
& e_{\xi \xi}=\frac{1}{2}\left[\left(g \cos \beta+\eta \Omega^{\prime}\right)^{2}+(g \sin \beta)^{2}-\frac{\left({ }^{0} \rho+\eta\right)^{2}}{{ }^{0} \rho^{2}}\right], \\
& e_{\xi \eta}=\frac{1}{2} g \sin \beta \ldots \ldots \ldots \ldots \ldots \ldots \ldots \ldots \ldots \ldots \ldots \ldots \ldots \ldots \ldots \ldots \ldots \ldots \ldots \ldots \ldots \ldots \ldots \ldots \ldots \ldots
\end{aligned}
$$

Here, consider another set of strain components $\varepsilon_{\xi \xi}$ and

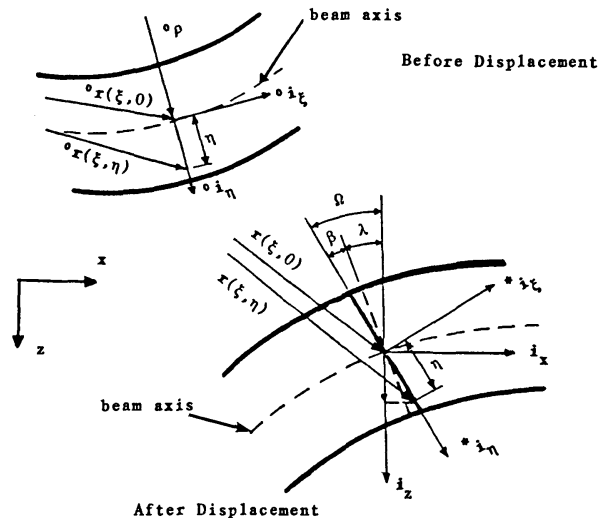

Fig. 1 Geometry of Curved Timoshenko Beam before and after Displacement 
$\varepsilon_{\xi \eta}$ which will be employed in the following constitutive relation. Let ${ }^{*} i_{\xi}$ and ${ }^{*} i_{\eta}$ denote the unit vectors in the direction normal and parallel to displaced cross-section (Fig. 1). Note that the infinitesimal element after displacement can be described in the directions of ${ }^{*} i_{\xi}$ and ${ }^{*} i_{\eta}$ as

$$
d \boldsymbol{r}(\xi, \eta)=\left\{g \cos \beta+\eta \Omega^{\prime}\right\} d \xi^{*} i_{\xi}+\{g \sin \beta d \xi+d \eta\}^{*} i_{\eta}
$$

From Eqs. (1), (2), and (6), defining the extensional strain $\varepsilon_{\xi \xi}$ as the difference between the magnitude of infinitesimal element in the derection normal to cross-section before and after displacement leads to

$$
\varepsilon_{5 \xi}=\frac{{ }^{0} \rho}{{ }_{0}^{0}+\eta}\left[\varepsilon+\eta\left(x-\frac{1}{{ }_{0}^{0} \rho}\right)\right] \text { in which } \varepsilon=g \cos \beta-1, \quad x=\Omega^{\prime} .
$$

In addition, defining the shear angle $\varepsilon_{\xi \eta}$ as the physical component of strain tensor $e_{\xi \eta}^{7)}$ results in $\varepsilon_{\xi \eta}=\frac{{ }^{0} \rho}{2\left({ }^{0} \rho+\eta\right)} \gamma$ in which $\gamma=g \sin \beta$

It is emphasized that, by the above definition of the new strain components, $\varepsilon_{\xi \xi}$ is a linear function of $\eta$, and $\varepsilon_{\xi \eta}$ is a uniform function of $\eta$ in case of the straight beam formulation.

\section{(2) Equilibrium Equations}

If the body and the end surface force vectors are denoted by $\boldsymbol{p}_{d}$ and $\boldsymbol{p}_{s}$, respectively, the virtual work equation for a curved beam element, employing the Kirchhoff stress tensors $\sigma_{i j}$ and the Green strain tensors $e_{i j}$, can be written by

$$
\int_{0}^{L} \int_{A}\left[\left\{\sigma_{\xi \xi} \delta e_{\xi \xi}+2 \sigma_{\xi \eta} \delta e_{\xi \eta}\right\}+\left\{\boldsymbol{p}_{d}{ }^{T} \delta \boldsymbol{r}(\xi, \eta)\right\}\right] \frac{0}{\rho}+\eta^{0} \rho d A d \xi+\left[\int_{A}\left\{\boldsymbol{p}_{s}{ }^{T} \delta \boldsymbol{r}(\xi, \eta)\right\} d A\right]_{\xi=0, L}=0
$$

Recalling that stress vector $\sigma_{\xi}$ acting on plane normal to $\xi$-axis is decomposed as $\sqrt{{ }^{0} \boldsymbol{g}_{\xi}^{0} \boldsymbol{g}_{\xi}}\left(\sigma_{\xi \xi} \boldsymbol{g}_{\xi}+\sigma_{\xi \eta} \boldsymbol{g}_{\eta}\right)$ in terms of orthogonal curvilinear coordinate ${ }^{7}$, an alternative decomposition of $\sigma_{\xi}$ to the new stress components $f_{\xi \xi}$ and $f_{\xi \eta}$ in the direction of ${ }^{*} i_{\xi}$ and ${ }^{*} i_{\eta}$, respectively, results in the relations as

$$
f_{\xi \xi}=\sigma_{\xi \xi} \frac{{ }^{0} \rho+\eta}{{ }^{0} \rho}\left\{g \cos \beta+\eta \Omega^{\prime}\right\}, \quad f_{\xi \eta}=\frac{{ }^{0} \rho+\eta}{{ }^{0} \rho}\left\{\sigma_{\xi \xi}(g \sin \beta)+\sigma_{\xi \eta}\right\}
$$

From the new definition of stress and strain components as given by Eqs. (7) and (9) it can be shown that

$\sigma_{\xi \xi} \delta e_{\xi \xi}+2 \sigma_{\xi \eta} \delta e_{\xi \eta}=f_{\xi \xi} \delta \varepsilon_{\xi \xi}+2 f_{\xi \eta} \delta \varepsilon_{\xi \eta}$

Substituting Eqs. (3) and (10) into Eq. (8) and remembering Eqs. (4) and (7) give

$\int_{0}^{L}\left(N \delta \varepsilon+M \delta x+V \delta \gamma+p_{x} \delta x+p_{z} \delta z+c \delta \Omega\right) d \xi+\left[P_{x} \delta x+P_{z} \delta z+C \delta \Omega\right]_{\xi=0, L}=0$.

where

$$
\begin{aligned}
& N=\int_{A} f_{\xi \xi} d A, \quad V=\int_{A} f_{\xi \eta} d A, \quad M=\int_{A} f_{\xi \xi} \eta d A \\
& p_{x}=\int_{A} p_{d x} \frac{{ }^{0} \rho+\eta}{0} d A, \quad p_{z}=\int_{A} p_{d z} \frac{{ }^{0} \rho+\eta}{{ }^{0} \rho} d A, \quad P_{x}=\int_{A} p_{s x} d A, \quad P_{z}=\int_{A} p_{s z} d A, \\
& c=\int_{A}\left[p_{d x} \cos \Omega-p_{d z} \sin \Omega\right] \eta \frac{{ }^{0} \rho+\eta}{{ }^{0} \rho} d A, \quad C=\int_{A}\left[p_{s x} \cos \Omega-p_{s z} \sin \Omega\right] \eta d A
\end{aligned}
$$

Then, the virtual work equation (11) for a one dimensional curved beam yields the equilibrium equations $(N \cos \Omega+V \sin \Omega)^{\prime}+p_{x}=0$

$(-N \sin \Omega+V \cos \Omega)^{\prime}+p_{z}=0$

$N \sin \beta-V \cos \beta+\frac{M^{\prime}+c}{g}=0$

and the boundary conditions at $\xi=0, L$ as

$$
\begin{array}{lll}
x={ }^{8} x & \text { or } & n(N \cos \Omega+V \sin \Omega)=P_{x} \\
z={ }^{g} z & \text { or } & n(-N \sin \Omega+V \cos \Omega)=P_{z} \\
\Omega={ }^{g} \Omega & \text { or } & n M=C \ldots \ldots \ldots \ldots \ldots \ldots \ldots \ldots \ldots \ldots \ldots \ldots \ldots \ldots \ldots \ldots
\end{array}
$$


where ${ }^{g} x,{ }^{g} z$ and ${ }^{g} \Omega$ are the given position components at the boundaries, and $n=-1,+1$ at $\xi=0, L$.

Note that the equilibrium equations (14) and boundary conditions (15) are identical to those by Reissner $^{1)}$, and Iwakuma et $\mathrm{al}^{4)}$. for straight beams. These results can also be applied to the problem of plane curved beams without shear deformation ${ }^{8,9)}$ by omitting the shear kinematic terms $\left(\beta, \varepsilon_{\xi \eta}\right)$.

\section{(3) Constitutive Equations}

For a linear elastic material, the simplest form of constitutive equations can be assumed by employing Young's modulus $\mathrm{E}$, and shear modulus $\mathrm{G}$ as

$$
f_{\xi \xi}=E \varepsilon_{\xi \xi}, f_{\xi \eta}=2 G \varepsilon_{\xi \eta}
$$

By selecting the $\xi$-coordinate along the centroidal axis, substituting Eq. (16) to Eqs. (12) yields the relations between stress and strain resultants expressed by

$$
N=E A \varepsilon, \quad M=E I\left(x-1{ }^{\circ} \rho\right), \quad V=G k A \gamma
$$

where

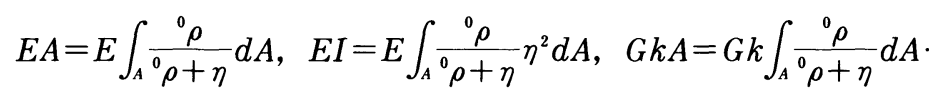

and the shear coefficient $k$ is newly introduced to reflect non-uniformly distributed shear deformation across the section ${ }^{10)}$.

It may be worth to note that, partly due to the ambiguity between the experimental and theoretical constitutive relations in beam theory, the utilization of physical stress $f_{i j}$ and strain components $\varepsilon_{i j}$ in Eq. (16), which simplifies the expression of governing equation, may be acceptable.

\section{FORMULATION OF STIFFNESS EQUATION OF PLANE STRAIGHT BEAM ELE- MENT}

As mentioned before, due to the complication of a curved beam element in FEM procedure, the following stiffness equation is developed for a straight beam element. By neglecting the effect of initial curvature $\left(1 / /^{0} \rho=0\right)$, the governing equation previously derived can be employed to formulate the nonlinear stiffness equation of a Timoshenko straight beam element in terms of nodal positions and rotations.

Select the first and third order polynomial functions for interpolation functions of $x$ and $z$, respectively. Taking into account that the zero-th order polynomial function or constant was properly selected for the interpolation function of shear angle $\beta$ in the formulation of the linear stiffness equation of Timoshenko beams ${ }^{11}$, this natural selection may also be used to derive the nonlinear stiffness equation.

In the view of finite element method, the length of each element is short enough to assume the base vector in the direction of beam axis at displaced state $i_{\xi}$ to be constant within element. For a particular element, choose $\boldsymbol{i}_{x}$ of arbitrary coordinate $(x, z)$ as shown in Fig. 2 such that

$$
i_{\xi} \cdot i_{x} \approx 1
$$

where $i_{\xi}$ can be expressed by

$$
\boldsymbol{i}_{\xi}=\left(x^{\prime} / g\right) i_{x}+\left(z^{\prime} / g\right) i_{z}
$$

Substituting Eq. (20) into Eq. (19) leads to

$\left(x^{\prime} / g\right) \approx 1,\left(z^{\prime} / x^{\prime}\right)^{2} \ll 1$

By taking into account of the above interpolation functions and the conditions of Eq. (21), substituting Eqs. (4), (7), and (17) into the virtual work equation (11), neglecting distributed forces, results in

$$
\delta \boldsymbol{x}^{t}\left[\boldsymbol{K} \cdot \boldsymbol{x}-\boldsymbol{F}-\boldsymbol{F}_{0}\right]=0
$$

where

$$
\begin{aligned}
\boldsymbol{K} & =\boldsymbol{K}_{E N}+\boldsymbol{K}_{E M}+\boldsymbol{K}_{G N}+\boldsymbol{K}_{G M} \\
\boldsymbol{x} & =\left[\begin{array}{lllllll}
x_{i} & z_{i} & \Omega_{i} \hat{L} & x_{j} & z_{j} & \Omega_{j} \hat{L} & \beta \hat{L}
\end{array}\right]^{t} \\
\boldsymbol{F} & =\left[\begin{array}{lllllll}
P_{x i} & P_{z i} & C_{i} / \hat{L} & P_{x j} & P_{z j} & C_{j} / \hat{L} & 0
\end{array}\right]^{t}, \hat{L}=x_{j}-x_{i}
\end{aligned}
$$

and the details of $\boldsymbol{K}_{E N}, \boldsymbol{K}_{E M}, \boldsymbol{K}_{G N}, \boldsymbol{K}_{G M}$, and $\boldsymbol{F}_{0}$ are given in Appendix. 


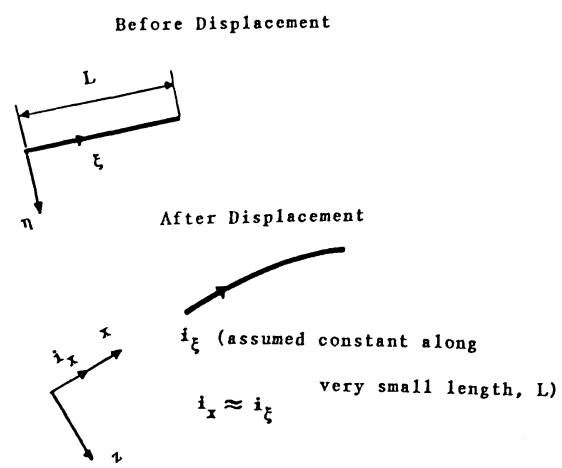

Fig. 2 One-Dimensional Straight Beam Element.

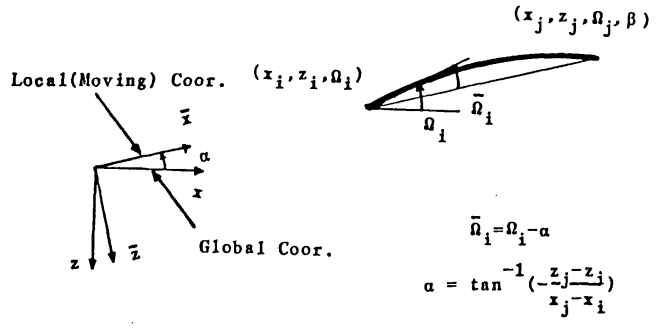

Fig. 3 Transformation of Coordinate at Equilibrium State.

The condition that Eq. (22) holds for arbitrary $\delta x$ is

$$
\boldsymbol{F}=\boldsymbol{K} \cdot \boldsymbol{x}-\boldsymbol{F}_{0}
$$

Eq. (24) is the nonlinear stiffness equation of a plane straight Timoshenko beam element with respect to the local coordinate system which satisfies Eq. (19). Note that, due to coupling terms of shear angle $\beta$, an independent nodal component $\beta$ has to be introduced for each element, In case of straight beam element without shear deformation, the stiffness equation (24) can be employed by neglecting the shear angle and shear rigidity terms $(\beta=0, G k A=\infty)$.

It is noted that the local coordinate identical to the so-called moving coordinate which has been employed to derive the element stiffness equation (24) must satisfy the condition of Eq. (19). Hence, in order to obtain the transformation of coordinates, the transformation of rotational angle has to be taken into account as shown in Fig. 3.

\section{NUMERICAL PROCEDURE}

Due to the nonlinearity of the stiffness equation, an iteration procedure is necessary for obtaining numerical solutions. A Newton-Raphson procedure was selected for iteration scheme. For general numerical treatment, the external force vector $F$ is expressed by the product of constant vector $f$ and variable scalar $f$, which are called loading pattern and loading intensity, respectively. Hence, denoting the incremental loading intensity and nodal positions between two iteration steps as $\Delta f^{n}$ and $\Delta x^{n}$, respectively, the numerical scheme for the stiffness equation can be written as

$$
\boldsymbol{K}_{T}\left(\boldsymbol{x}^{n}\right) \Delta \boldsymbol{x}^{n}-\Delta \boldsymbol{f}^{n} \boldsymbol{f}=f^{n} \boldsymbol{f}-\left[\boldsymbol{K}\left(\boldsymbol{x}^{n}\right) \boldsymbol{x}^{n}-\boldsymbol{F}_{\mathbf{0}}\left(\boldsymbol{x}^{n}\right)\right]
$$

where $K_{T}(x)$ denotes the tangent stiffness matrix. Since it was theoretically proved that the total stiffness matrix $\boldsymbol{K}(\boldsymbol{x})$ is approximately equal to tangential stiffness matrix $\boldsymbol{K}_{T}(\boldsymbol{x})^{6)}, \boldsymbol{K}(\boldsymbol{x})$ is employed instead of $\boldsymbol{K}_{T}(\boldsymbol{x})$ in Eq. (25). Also, numerical proof of this approximation is presented in the next section.

Since the number of unknowns is larger by one than that of the stiffness quation, an additional condition must be introduced among variables $\Delta x$ and $\Delta f$. In this treatment, two techniques for controlling these variables are utilized. The first is the load control technique, which can be expressed in the form of ${ }^{(5), 9)}$

$\Delta f^{n}-c=0$

where $c$ is a constant parameter for obtaining the next solution.

And the second is the path length control technique in $(j+1)$-dimension space, which is given by ${ }^{5) .9)}$

$\sum_{i=1}^{j} a_{i}^{2}\left(x_{i}-x_{i}^{0}\right)^{2}+a_{j+1}^{2}\left(f-f^{0}\right)^{2}-c^{2}=0$

\section{NUMERICAL EXAMPLES}

The first example is a cantilever beam under a transverse tip load in Fig. 4. Also shown in Fig. 4 is the analytical finite displacement solution of beam without shear deformation by Mattiasson ${ }^{12}$. The present 


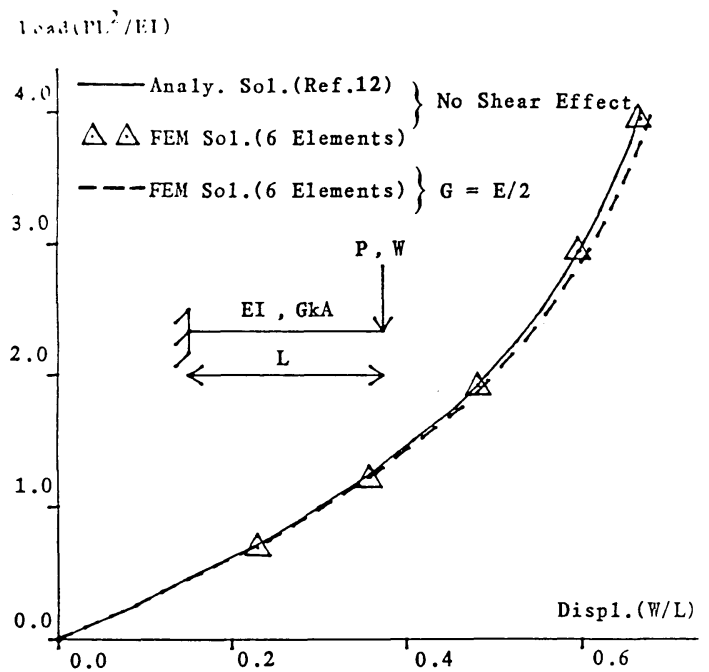

Fig. 4 Results of Cantilever Beam under a Transverse Tip Load.

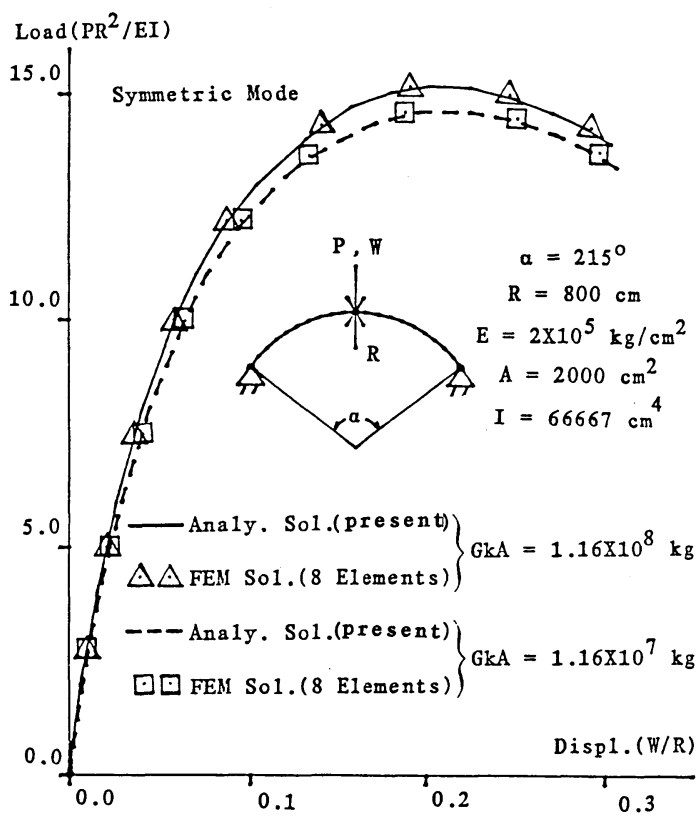

Fig. 5 Results of Circular Two-Hinged Arch under a Point Load.

solution obtained by the stiffness equation of Eq. (24) neglecting shear deformation agrees well with the analytical solution. In order to show the validity of the stiffness equation of beam with shear deformation (Eq. 24), this problem is calculated by assuming shear modulus equal to one half of Young's modulus. There exists some difference between this result and the analytical result ${ }^{12)}$ due to the effect of shear deformation.

The second is a symmetrical circular two-hinged arch under a point load at crown as shown in Fig. 5. The present analytical solutions by Runge-Kutta-Gill Method for Eqs. (14) and (15) are given in Fig. 5 for two different values of shear stiffness, and compared with the FEM solutions obtained by Eq. (24). The results show a good coincidence between the finite element solutions of a series of straight beams and the analytical solutions of the curved beam.

Lastly, Fig. 6 shows the present FEM solutions for the relation between a point load and deflection at the crown of unsymmetrical circular clamped-hinged arch, in which the effect of shear deformation is neglected. The present results are compared with the results of Yoshida et $\mathbf{a l}^{13)}$. The first equilibrium path starting from the origin point to point $\mathrm{A}$ is in good agreement with the results by Yoshida. Yoshida also found another equilibrium path starting from point $B$. However, he did not compute any numerical results between point $A$ and point $B$, but only connected them by dash line shown in Fig. 6 . By using the path length control method in this study, it is found that point $\mathrm{A}$ and point $\mathrm{B}$ lie on the different equilibrium path. In order to start tracing the second equilibrium path, it is noted that the equilibrium configuration of arch at point $\mathrm{B}$ obtained by Yoshida is employed for initial guess solution, and the displacement in the direction of applied load at crown is prescribed in the iterative procedure. The equilibrium configurations at different states are shown in Fig. 7.

Fig. 6 also includes the relation between displacement and the smallest eigenvalue determined from the total stiffness matrix $K(x)$ along the first equilibrium path. It can be seen that the line of eigenvalue in the range of both limit points $\mathrm{D}$ and $\mathrm{C}$ passes the horizontal axis, i. e., zero eigenvalue line. From the fact that the tangential stiffness matrix $K_{T}(x)$ becomes singular at limit point, the deviation of the limit point and the point of zero eigenvalue shows the error due to the approximation between $K_{T}(x)$ and $\boldsymbol{K}(\boldsymbol{x})$. In order to 


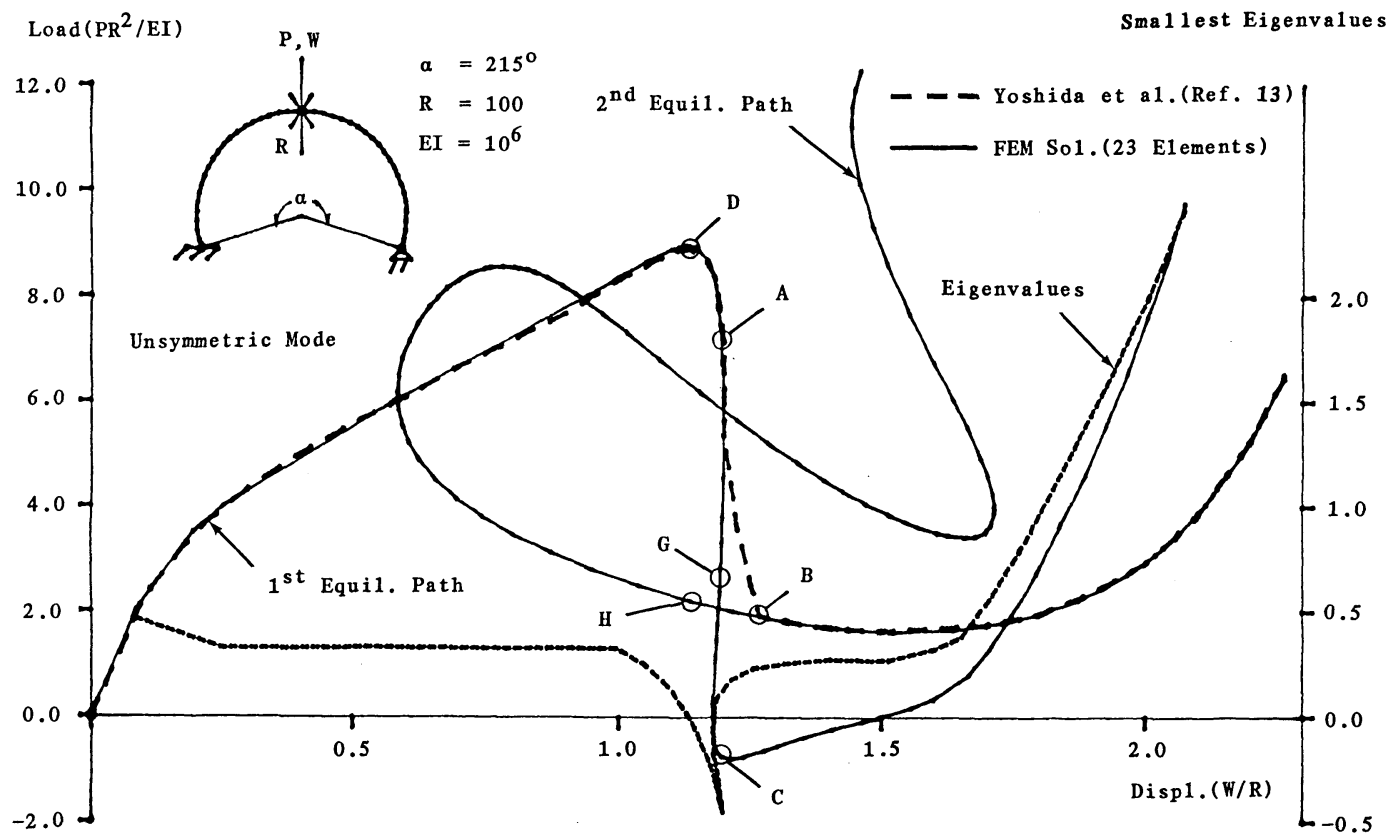

Fig. 6 Results of Circular Clamped-Hinged Arch under a Point Load.
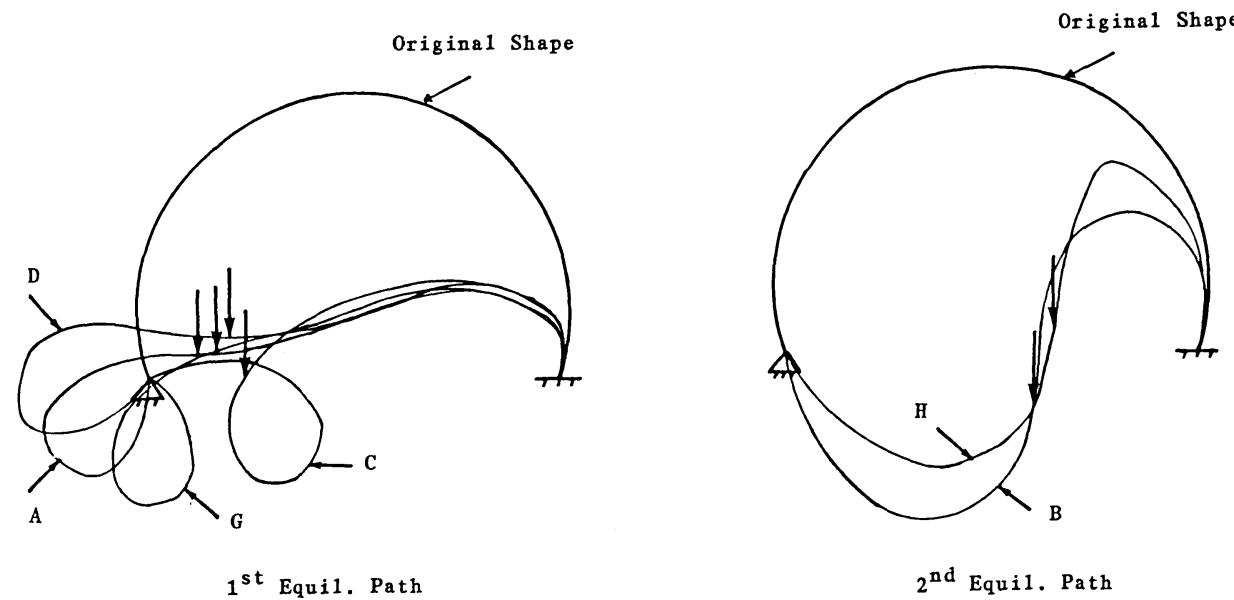

Fig. 7 Equilibrium Configurations of Circular Clamped-Hinged Arch.

check this error numerically, the difference of displacement (corresponding to applied load) at the limit points and at the zero eigenvalue points are calculated, and the values are less than $1 \%$ and $3 \%$ in the range of points $\mathrm{D}$ and $\mathrm{C}$, respectively.

\section{CONCLUDING REMARKS}

This study formulates an analysis of elastic finite displacements of plane curved Timoshenko beams, with rigorous consistency of kinematics through the application of the virtual work equation. The adoption of stress and strain components defined in Eqs. ( 7 ) and (9) and the constitutive equations (16) makes the expression of the governing equation much simpler and easier to solve compared with the governing equation in Ref. 2.

By utilizing a total Lagrangian approach, the finite displacement stiffness equation of an elastic plane 
straight beam with and without shear deformation is presented in terms of nodal forces and positions. As shown in the second example, the stiffness equation of a plane straight beam element in this study is applicable for an arbitrary plane curved beam by dividing a curved beam into a series of straight beam elements. In the last example, it has been checked numerically that, with the selection of local coordinates along the displaced beam axis, the approximation between the total stiffness matrix in Eq. 24 and the tangential stiffness matrix in Eq. 25 is acceptable. Therefore, the tangential stiffness matrix can be replaced by the total stiffness matrix in Newton-Raphson procedure. From the viewpoint of numerical analysis, a path length control technique seems to be an effective tool to follow the equilibrium path.

\section{ACKNOWLEDGEMENT}

This research is supported in part by the Grant-in-Aid for Scientific Research provided from the Japanese Ministry of Education, Science and Culture.

\section{APPENDIX. STIFFNESS MATRICES}

The detailed forms of stiffness matrices appeared in Eq. (23) are given below

$$
\begin{aligned}
& \boldsymbol{K}_{E N}=(E A / L)+(G k A-E A) \beta^{2} / L\left[\begin{array}{ccccccc}
1 & 0 & 0 & -1 & 0 & 0 & 0 \\
& 0 & 0 & 0 & 0 & 0 & 0 \\
& & 0 & 0 & 0 & 0 & 0 \\
& \text { Sym. } & & 1 & 0 & 0 & 0 \\
& & & & 0 & 0 & 0 \\
& & & & & 0 & 0 \\
& & & & & & 0
\end{array}\right] \\
& \boldsymbol{K}_{E M}=E I /\left(L \hat{L}^{2}\right)\left[\begin{array}{ccccccc}
B_{1} & 0 & 0 & -B_{1} & 0 & 0 & 0 \\
& 12 & -6 & 0 & -12 & -6 & 12 \\
& & 4 & 0 & 6 & 2 & -6 \\
& \text { Sym. } & & B_{1} & 0 & 0 & 0 \\
& & & & 12 & 6 & -12 \\
& & & & & 4 & -6 \\
& & & & & & 12+(1 / D)
\end{array}\right] \\
& \boldsymbol{K}_{G N}=P / \hat{L}\left[\begin{array}{ccccccc}
B_{2} & 0 & 0 & -B_{2} & 0 & 0 & 0 \\
& 6 / 5 & -1 / 10 & 0 & -6 / 5 & -1 / 10 & 1 / 5 \\
& & 2 / 15 & 0 & 1 / 10 & -1 / 30 & -1 / 10 \\
& \text { Sym. } & & B_{2} & 0 & 0 & 0 \\
& & & & 6 / 5 & 1 / 10 & -1 / 5 \\
& & & & & 2 / 15 & -1 / 10 \\
& & & & & & -4 / 5
\end{array}\right]
\end{aligned}
$$

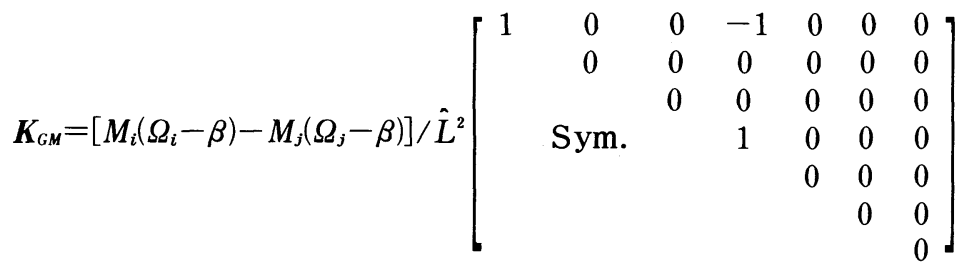

$$
\boldsymbol{F}_{0}=E A\left\{1-\left(\beta^{2} / 2\right)\right\}\left[\begin{array}{lllllll}
-1 & 0 & 0 & 1 & 0 & 0 & 0
\end{array}\right]^{t}
$$

where

$$
\begin{aligned}
& \hat{L}=x_{j}-x_{i}, \quad D=E I /\left(G k A \hat{L}^{2}\right), P=E A(\hat{L}-L) / L \\
& B_{1}=4\left(\Omega_{i}^{2}+\Omega_{i} \Omega_{j}+\Omega_{j}^{2}+3 \beta^{2}-3 \Omega_{i} \beta-3 \Omega_{j} \beta\right), \quad B_{2}=\left(2 \Omega_{i}^{2}-\Omega_{i} \Omega_{j}+2 \Omega_{j}^{2}+3 \beta^{2}-3 \Omega_{i} \beta-3 \Omega_{j} \beta\right) / 15 L \\
& M_{i}=\left\{-2 E I\left(2 \Omega_{i}+\Omega_{j}\right) / L\right\}+6 E I \beta / \hat{L}, \quad M_{j}=\left\{2 E I\left(2 \Omega_{i}+\Omega_{j}\right) / L\right\}-6 E I \beta / \hat{L}
\end{aligned}
$$




\section{REFERENCES}

1) Reissner, E. : On One-Dimension Finite-Strain Beam Theory : the Plane Problem, Journal of Applied Mathematics and Physics (ZAMP), Vol.23, 1972.

2) Sheinman, I. : Large Deflection of Curved Beam with Shear Deformation, Journal of the Engineering Mechanics Division, ASCE, Vol. 108, No. EM 4, Proc. Paper 17258, Aug., 1982.

3) Taweep, C., Naotsugu, S., Tan K. H. : Discussion on Large Deflection of Curved Beam with Shear Deformation by Sheinman, I. (Paper 17258), Journal of the Engineering Mechanics Division, ASCE, Vol. 109, No. EM 5, Aug., 1983.

4) Iwakuma, T., and Kuranishi, S. : How much contribution does the Shear Deformation Have in a Beam Theory, Proc. of JSCE, No. 344/ I-1 (Struct. Eng. /Earth. Eng.), Apr., 1984.

5) Nishino, F., Ikeda, K., Sakurai, T., and Hasegawa, A. : A Total Lagrangian Nonlinear Analysis of Elastic Trusses, Proc. of JSCE., No. 344/ I-1, 1984.

6) Taweep, C. : An Elastic Finite Displacement Analysis of Inplane Beams with Shear Deformation, a Master Thesis at the University of Tokyo, Dept. Civil Engrg., Japan, Sep., 1984.

7) Fung. Y.C., Foundation of Solids Mechanics, Prentice-Hall, Inc., 1965.

8) Ai, M., and Nishino F. : Geometrically Nonlinear Mechanics in Discrete System and Application to Plane Frame-Works, Journal the Faculty of Engineering, the University of Tokyo (B), Vol. xxx vi, No. 4, 1982.

9) Nishino, F., Mechanics of Continua (II), Structural Mechanics, Doboku Kogaku Taikei (Edited by Y. Takahashi), Vol.6, Shokoku-Sha Pub. Co., Tokyo, 1984, (in Japanese).

10) Cowper, G. R. : The Shear Coefficient in Timoshenko's Beam Theory, J. Appl. Mech., Vol. 33, 1966.

11) Iwakuma, T., Ai, M., and Nishino, F. : On Derivation of Timoshenko Beam Stiffness Equation, Proc. of JSCE, No. 312 , Aug., 1981.

12) Mattiasson, K. : Numerical Results from Large Deflection Beam and Frame Problems Analysed by Means of Elliptic Intregrals, Int. J. Num, Meth, Engng, 17, 1981.

13) Yoshida, Y., Masuda, N., Morimoto, T., and Hirosawa, N. : An Incremental Formulation for Computer Analysis of Spaced Framed Structures, Proc. of JSCE., No. 300, Aug., 1980, (in Japanese).

14) Hildebrand, F.B., Introduction to Numerical Analysis, McGraw-Hill, 1974.

15) Nishino, F. and Hasegawa, A. : Thin-Walled Elastic Members, Journal of the Faculty of Engineering, the University of Tokyo (B), Vol. $x x x v$, No.2, 1979.

16) Timoshenko, S. P., and Goodier, J. N., Theory of Elasticity, McGraw-Hill Book Co., New York, 1970.

(Received August 21 1985) 